\title{
Traduire
}

Ine eutre perspective sur I t tataduction

Revue française de la traduction

$230 \mid 2014$

À la croisée du texte et de l'image

\section{Les principaux aspects pratiques de la mission d'interprétation consécutive officielle}

\section{Gilles Ouvrard}

\section{(2) OpenEdition}

1 Journals

Édition électronique

URL : http://journals.openedition.org/traduire/639

DOI : $10.4000 /$ traduire.639

ISSN : 2272-9992

Éditeur

Société française des traducteurs

Édition imprimée

Date de publication : 15 juin 2014

Pagination : 108-125

ISSN : 0395-773X

\section{Référence électronique}

Gilles Ouvrard, «Les principaux aspects pratiques de la mission d'interprétation consécutive

officielle », Traduire [En ligne], 230 | 2014, mis en ligne le 15 juin 2016, consulté le 10 décembre 2020.

URL : http://journals.openedition.org/traduire/639 ; DOI : https://doi.org/10.4000/traduire.639 


\section{Les principaux aspects pratiques de la mission d'interprétation consécutive officielle}

\section{Gilles Ouvrard}

Dans le précédent numéro de Traduire(1), après une introduction générale, nous avons traité des circonstances précédant la mission d'interprétation officielle, en terminant par les aspects concernant le recrutement.

Dans cette seconde partie, nous parlerons de la réalisation de la mission, de sa préparation d'abord, puis de son déroulement. L'interprétation officielle présente la particularité de s'exercer en des lieux qui changent en permanence, ce qui implique des déplacements parfois lointains, et la nécessité de les effectuer en limitant le plus possible leur incidence sur la qualité de la prestation elle-même. Nous allons en évoquer les principaux aspects pratiques, concernant la logistique personnelle, puis certains aspects spécifiques des conditions de travail, tels que les cortèges, la sécurité, la gestion du stress et du trac, de la fatigue, etc. Nous évoquerons ensuite les relations entre l'interprète et les personnes présentes, puis dirons quelques mots du placement de l'interprète dans les différentes circonstances du cours de la mission, avant de conclure sur une situation particulière marquant la fin de celle-ci.

\section{La préparation de l'interprète}

Tout interprète doit être un " honnête homme " de son siècle, et se tenir informé de l'actualité internationale. Pour l'interprétation officielle, l'interprète doit de plus bien connaître les institutions des deux pays dont il pratique les langues, et en suivre les dossiers importants, d'actualité ou de fond ; sur le plan linguistique, il doit connaître et pratiquer le langage diplomatique dans toutes ses nuances, comme il doit maîtriser le registre particulier des discours protocolaires, avec leurs références culturelles et expressions fleuries(2).

(1) Le présent texte est la suite de l'article intitulé L'Interprétation consécutive officielle publié dans Traduire ${ }^{\circ} 229$.

(2) Même si souvent ce type de discours fait l'objet d'une traduction écrite préalable qu'il lit en séance. 
II s'agit là d'une préparation générale, permanente, pratiquée de manière habituelle par l'interprète, qu'il ait ou non une mission en vue. Elle lui permet de mettre à jour et développer ses connaissances chaque fois qu'il rencontre une information utile.

Il existe par ailleurs une préparation spécifique : la préparation de la mission pour laquelle l'interprète est recruté. Cette préparation-ci est ciblée, et couvre l'ensemble des sujets susceptibles d'être abordés pendant ladite mission. Elle vise à collecter les informations disponibles, dans les deux langues, concernant les sujets prévus.

Pour ces deux temps de préparation, le développement d'internet a grandement facilité la tâche de l'interprète. Ces recherches à partir de sources publiques sont aujourd'hui devenues très banales, nous ne nous y attarderons pas.

J'insisterai cependant sur un point : les listes de vocabulaire que l'on établit ne servent par ellesmêmes à rien, ce qui importe c'est que leur contenu soit présent et disponible sans effort au moment où l'interprète en aura besoin. Elles doivent être efficacement mémorisées, ce qui nécessite de les réviser, avec une grande concentration.

Dans ma propre pratique, j'avais pris l'habitude d'élaborer des listes " très larges ", comprenant, outre d'éventuels termes spécialisés, le vocabulaire et les tournures ou expressions propres à la diplomatie et qui, n'étant pas d'un usage quotidien, ont besoin d'être réactivés. Ces listes provenaient de missions antérieures sur le même sujet, enrichies par la préparation du moment ; je les révisais régulièrement avant et pendant la mission. II s'agissait de listes manuscrites, ce point est important pour moi. Le fait de les écrire soi-même à la main et non de les imprimer automatiquement à partir d'un fichier permet, si l'on est concentré, de retenir plus efficacement le vocabulaire. Ces listes manuscrites sont par ailleurs chargées de souvenirs qui reviennent en mémoire lorsqu'on les révise, et contribuent à faciliter la préparation.

Outre les sources publiques auxquelles il se réfère, l'interprète utilise également d'autres documents, qui servent à finaliser la préparation. Ces documents sont officiels, mais non publics. L'interprète n'en dispose généralement que très peu de temps avant la mission, et souvent seulement pendant celle-ci.

\section{Les documents officiels}

Ces documents officiels de diffusion restreinte sont de trois types : le dossier de présentation de la visite, le "livret " de la visite et les "éléments de langage".

Habituellement produit par les services de l'ambassade de France, le dossier présentant la visite, souvent volumineux (ce qui est un problème pour les bagages), est remis à leur arrivée dans le pays aux participants français à une mission à l'étranger. II retrace le cadre de la visite, décrit le programme, donne des informations pratiques ou culturelles sur les endroits qui seront visités, des informations bibliographiques sur les personnalités qui seront rencontrées. Il ne s'agit que d'informations générales. 
Le "livret " est un petit carnet tenant dans la poche qui contient toutes les indications pratiques concernant la visite : liste détaillée de la délégation, nature, lieu et horaire des manifestations, liste des personnes concernées, tenue à porter (de ville, ou de soirée), composition des cortèges de voitures, rendez-vous pour la prise en charge des bagages, etc.(3). Toutes les activités de la personnalité principale (par exemple le président de la République) y sont décrites par le menu, notamment à l'aide de schémas indiquant le trajet précis du parcours emprunté par la personnalité, et la position des différents participants. C'est, sur le plan logistique cette fois, un document de travail indispensable pour l'interprète, tant pour la préparation que pour l'exécution de sa mission. II doit absolument en disposer, et le réclamer au service du Protocole si on oublie de le lui communiquer.

Enfin, les " éléments de langage ", selon la terminologie française, sont les notes aide-mémoire préparées spécifiquement par les conseillers pour la personnalité en vue de la rencontre qui va avoir lieu. Ce document précise donc les thèmes prévus, mais fait également le point sur l'état d'un dossier, indique l'appréciation de la situation par la partie française, rappelle la position officielle sur la question, propose l'angle sous lequel le dossier peut être abordé, et la manière d'en parler. Bien entendu, la personnalité reste entièrement libre de ses propos, notamment en ce qui concerne les questions de principe et les problèmes politiques proprement de son ressort, mais dans la pratique j'ai pu constater qu'elle s'écartait rarement des lignes tracées par les "éléments de langage ". Sans doute parce que les dossiers, notamment industriels ou commerciaux, sont souvent très techniques, et que la personnalité n'en maîtrise pas les détails : elle doit se fier à un guide. Elle a donc les éléments de langage à portée de la main pendant les entretiens et les consulte discrètement pendant les temps d'interprétation vers la langue étrangère, en préparation de la suite de son intervention.

II faut préciser qu'il n'est pas facile, sinon de se faire communiquer, au moins de consulter les éléments de langage, parce que ceux-ci sont parfois mis au point tardivement, mais également parce qu'ils sont extrêmement confidentiels. II est donc fréquent que l'interprète ne puisse en prendre connaissance que quelques minutes avant le début de la séance. Quoi qu'il en soit, leur consultation est très importante, et sans assiéger les personnes concernées, il ne faut pas hésiter à insister courtoisement auprès d'elles pour en prendre - ne serait-ce que très rapidement - connaissance. Même si son exploitation est très tardive, c'est LE document essentiel pour finaliser la préparation, en appréhendant les derniers éléments qui auraient pu y échapper.

(3) Les grandes visites bilatérales font l'objet par les services compétents d'une préparation extrêmement poussée visant à éviter toute surprise et tout incident. La visite officielle est ainsi précédée par une ou plusieurs missions de "précurseurs " appartenant notamment au service du Protocole, qui vont avec leurs homologues prévoir et organiser les moindres détails de son déroulement. C'est au terme de cette phase de préparation qu'est élaboré le livret en question. 
Nous allons maintenant parler des aspects du déroulement de la mission, en commençant par la logistique personnelle de l'interprète.

\section{La logistique personnelle}

\section{La forme physique}

De manière générale, l'interprète doit être en bonne forme physique, parce que ces missions sont toujours épuisantes, du fait du décalage horaire, de la tension, du rythme des activités, etc. II doit en particulier veiller dans les jours qui précèdent à ne rien faire qui risque de le handicaper(4).

Signalons au passage qu'inversement, en dépit de la fatigue, on tombe rarement malade en mission. Je n'ai été malade que deux fois en près de trente ans et me souviens avoir récupéré très rapidement dans les deux cas. D'une certaine manière, on ne peut pas "se le permettre". Mais bien sûr il est important de prendre en permanence des précautions, nous en donnerons un exemple plus loin.

\section{Les deux obligations fondamentales de l'interprète}

Encore étudiant à l'ESIT, je me souviens de cette remarque d'un de nos enseignants(5) : "On peut tout pardonner à un interprète, sauf d'être en retard et de ne pas avoir une mise correcte. "Ce n'était pas une boutade, mais un rappel de règles fondamentales s'agissant de toute prestation professionnelle. Ces deux aspects relèvent d'ailleurs l'un et l'autre de la " neutralité " nécessaire : l'interprète ne doit pas attirer l'attention sur sa personne. Ce qui est vrai d'une mission pour le privé l'est encore davantage pour une mission officielle.

\section{La ponctualité}

Sur le premier point, la ponctualité, une des clés réside dans l'anticipation. C'est ce que permet le repérage. Lorsque les circonstances le permettent, pour éviter toute surprise, il est souhaitable avant la mission d'effectuer un repérage sur place, pour vérifier exactement les temps de déplacement et l'accès aux lieux (salles, etc.). II est également indispensable de prévoir à chaque étape une marge de sécurité.

(4) Je me souviens m'être lors d'une activité privée l'avant-veille d'un départ fait un " tour de rein " tel que je ne pouvais même pas soulever ma valise ; je n'ai pu assurer ma mission que grâce à l'amabilité de notre confrère Bruno Gensburger, membre de l'équipe d'interprètes, qui a eu la gentillesse de s'occuper de mon bagage pendant tout le voyage.

(5) II s'agissait de Monsieur Emmanuel Weintraub. 


\section{La marge de sécurité}

Pour tout déplacement, lointain ou proche, il faut prévoir une grosse marge de sécurité, et arriver largement à l'avance sur place ou au moins à proximité. Le jour du départ par exemple, rien n'est plus perturbant que de se demander si on ne va pas arriver en retard au rendezvous parce que, ce jour-là, il y a eu un incident sur sa ligne de métro habituelle.

II ne faut pas oublier qu'outre le temps de déplacement personnel, par exemple dans les transports en commun, après l'arrivée à l'entrée d'un lieu officiel, il peut encore falloir plusieurs minutes pour parvenir à l'endroit convenu, notamment du fait des mesures de sécurité. II faut tenir compte du temps d'attente généré par le franchissement des sas de sécurité, dont la durée peut varier sensiblement.

\section{Le piège des ascenseurs}

Il faut également au cours de la mission se méfier de circonstances a priori banales, comme l'attente des ascenseurs dans les hôtels modernes tout en hauteur : aux heures " de pointe ", lorsque tout le monde quitte sa chambre, on peut se retrouver à attendre de longues minutes que l'ascenseur finisse par arriver... II vaut donc mieux descendre nettement à l'avance, puis se placer à un endroit commode pour surveiller l'arrivée de la délégation.

Le client ou ses services doivent savoir qu'il n'y a pas de souci, que l'on est bien arrivé. Pour les missions officielles, il faut ainsi se présenter au rendez-vous une demi-heure à l'avance.

Aujourd'hui, on doit aussi être joignable. Cela va de soi avec la généralisation des portables, mais rappelons que pour toute mission, longue ou courte, il est indispensable de s'être préalablement muni du numéro de téléphone et du nom d'un responsable, du Protocole ou d'un autre service, pour pouvoir prévenir en cas de problème(6).

\section{La tenue}

Quelle tenue faut-il porter ? La question se pose à la fois pour le travail lui-même et les déplacements. Les hommes ont l'avantage de faire face à toutes les situations ou presque avec la même tenue costume-cravate, alors que les femmes disposent d'un éventail de possibilités plus large. Correction (cela va de soi), élégance (pourquoi pas), mais aussi confort, commodité et, au final toujours la même recommandation : la sobriété(7).

(6) Il est conseillé lorsqu'on vous indique un numéro et un nom d'appeler la personne afin de vérifier qu'il n'y a pas eu erreur. Ne pas oublier par ailleurs de couper son portable quand on a rejoint la délégation.

(7) Un des impératifs est par ailleurs de ne pas se tacher, là encore dans le souci de ne pas attirer l'attention. Ce n'est pas si facile que cela pendant les repas, si l'on interprète en mangeant. 


\section{Les bagages}

Si l'on prend l'avion avec des bagages mis en soute, bien que le service des Voyages officiels soit très efficace, il est prudent de ne pas tabler sur leur disponibilité immédiatement à l'arrivée. Leur livraison dans les chambres prend forcément un peu de temps, et pour les déplacements de groupes importants, peut avoir lieu après l'horaire de début de la mission.

Pour ma part, j'ai donc toujours pour ces missions voyagé en costume, avec un bagage à main léger comportant tous les outils nécessaires (carnet de notes, stylos, etc.). De même, il est prudent de ne pas se séparer des documents importants tels que les discours (originaux et traductions)(8). Le temps de vol est d'ailleurs souvent mis à profit pour des mises au point encore parfois longues. Par contre, il vaut mieux ne pas s'encombrer de dossiers ou livres que l'on n'aura de toute façon pas le temps ni l'énergie de consulter.

Pendant toute la durée de la mission, en cas de prise en charge groupée des bagages, il convient également de rester attentif aux consignes qui sont diffusées par le service des Voyages.

\section{Aspects spécifiques des conditions de travail}

Nous allons maintenant parler de différents aspects spécifiques des conditions de travail.

\section{Les cortèges}

Par cortèges, il faut entendre les cortèges automobiles, qui transportent d'un site à l'autre la délégation, ou plus précisément " les " délégations( 9 ).

Dans le livret décrivant le voyage dont nous avons parlé plus haut sont indiqués précisément les numéros des véhicules (voitures ou car) que chacun doit emprunter (comprendre parfois " monter en catastrophe ") à chaque étape du voyage. À la descente de l'avion, au moment où commence vraiment la mission, tout en prenant place au pied de l'échelle à l'endroit où la personnalité qu'il accompagne va être accueillie par le représentant des autorités locales, l'interprète doit identifier rapidement par son numéro affiché derrière le pare-brise le véhicule dans lequel il va monter pour gagner ensuite l'hôtel : ce peut être le véhicule de la personnalité, au cas où une interprétation requiert sa présence ; ce peut être un autre véhicule dans le

(8) À la fin du vol, vérifier qu'on n'a rien fait tomber par terre ou laissé à sa place...

(9) Car il y a la délégation de la personnalité, la délégation des hommes d'affaires, et la délégation des journalistes, ces deux dernières voyageant souvent par des moyens collectifs comme les minibus ou les cars ; mais ici nous parlerons de la délégation de la personnalité, dans laquelle est habituellement intégré l'interprète. 
cas contraire. Les voitures changent parfois de place, et rien ne ressemble plus à une berline noire aux vitres fumées qu'une autre berline noire. II y a souvent une certaine confusion au moment de monter dans les véhicules. II est donc sage de vérifier qu'il n'y a pas eu de changement, et de se faire connaître du chauffeur, qui pourra faire signe si l'on cherche sa voiture.

La vitesse de déplacement des cortèges a été pendant longtemps très différente en France et en Chine(10). Autrefois dans les cortèges officiels chinois, les interprètes étaient très bien installés dans les grandes limousines Drapeau Rouge, qui comportaient deux strapontins face à la banquette arrière sur laquelle prenaient place les personnalités. Dans les véhicules plus récents, un seul interprète est assis à l'avant, légèrement tourné pour pouvoir parler en direction de l'arrière où se trouvent les personnalités.

Un danger redoutable contre lequel je me suis toujours systématiquement prémuni dans les voitures est celui de la climatisation. Dans le cas général où la personnalité est à l'arrière et l'interprète à l'avant, en été, la climatisation envoie l'air froid droit sur lui. Même si elle n'est pas très forte, il est très facile d'attraper froid en quelques minutes. Ma parade a consisté à me protéger avec ma sacoche posée sur mes genoux, faisant ainsi office de déflecteur écartant le flux d'air froid. J'avais par ailleurs toujours une écharpe légère dans ma sacoche, et je m'en suis parfois servi, même pour quelques minutes seulement.

\section{Les déplacements à pied}

La particularité des déplacements à pied de l'interprète, c'est qu'il doit toujours rester à proximité immédiate des personnalités pour lesquelles il interprète, pour bien entendre ce qu'elles disent, et qu'elles puissent aisément l'entendre. Ce n'est pas facile, car il y a en général beaucoup de monde autour des personnalités dans ces moments-là : les agents du protocole, de la sécurité, les gardes du corps, éventuellement la conjointe ou le conjoint, un ou des collaborateurs, les accompagnateurs locaux, et souvent des photographes et les caméras de télévision, etc. L'interprète doit rester dans le premier cercle, celui qui est au contact direct des interlocuteurs. II lui faut ainsi en permanence " coller " aux personnalités. Ceci nous amène à parler de la sécurité.

\section{La sécurité}

Nous venons de dire que pendant les déplacements au cours desquels il travaille, l'interprète doit rester au contact, mais il lui faut en même temps veiller à ne pas être trop près, et risquer

(10) Le déplacement des cortèges était rapide en France, plus lent en Chine, mais l'interprète a de toute façon intérêt à se prémunir contre un freinage imprévu, et à se tenir à la poignée située au-dessus de la porte. 
s'il y a un instant de bousculade de heurter la personnalité(11). C'est le cas notamment à l'arrivée dans les lieux étroits comme les couloirs, ou dans les moments où il y a changement de mode de déplacement, par exemple quand on prend un ascenseur. II faut " coller ", certes, mais prudemment.

En dehors des déplacements à pied, la sécurité est de manière générale un facteur très important dans ces missions.

Par sécurité, en fait j'entends ici la sécurité personnelle de l'interprète, mais aussi celle de la personnalité. II peut sembler curieux qu'outre celle de l'interprète lui-même, j'évoque la sécurité de la personnalité, entourée par des agents dont c'est précisément la mission. Mais les circonstances peuvent faire qu'à un moment donné ce soit l'interprète qui prenne le premier conscience d'un risque, et soit le seul à pouvoir réagir immédiatement. Bien que ce ne soit pas fondamentalement son rôle, selon moi cela fait tout à fait partie de sa prestation auprès de la personnalité. Dans les missions impliquant de fréquents déplacements, et même dans les salles de réunion, il existe nombre de chausse-trapes et pièges sournois : des câbles électriques au sol, des marches irrégulières, des passages étroits ou surbaissés(12), alors même que le déplacement s'effectue en groupe dans des conditions, comme on l'a vu, déjà difficiles. Cela ne m'est jamais rien arrivé personnellement, mais j'ai assisté à plusieurs incidents, des chutes notamment, et vu une personne de l'accompagnement heurtée au visage par une caméra. Ce qui rend les choses encore plus délicates, et ce pourquoi l'interprète doit rester particulièrement vigilant, c'est que son attention est largement mobilisée par son travail. II est en train d'interpréter, mais il doit cependant toujours rester conscient de tous les obstacles ou dangers qui peuvent se présenter, et les anticiper en permanence.

II existe encore, lors des voyages officiels, différents modes de déplacement autres que les cortèges automobiles, comme le train, l'avion, voire l'hélicoptère. Chaque fois qu'il est appelé à travailler pour la personnalité, l'interprète devra faire le nécessaire pour continuer à travailler efficacement, tout en respectant les règles de sécurité en vigueur : s'assoir correctement, attacher sa ceinture, etc. Cela semble encore une fois évident, mais on peut avoir tendance, par déférence pour la personnalité, à vouloir lui laisser le maximum de place, par exemple pour étendre ses jambes. Là encore, le principe applicable est d'avoir un comportement normal, respectant notamment les règles de sécurité.

\section{La gestion du stress et du trac}

L'interprète est sujet à l'un et l'autre de ces deux états, qu'il convient de distinguer. Je définirai le stress comme un état de tension permanent, résultant du fait d'assumer une responsa-

(11) Je me souviens ainsi avoir cogné le talon du président Mitterrand, qui n'avait rien dit mais avait dû avoir très mal.

(12) Ces particularités ont normalement été repérées par les précurseurs, mais il y a toujours une part d’imprévisible. 
bilité importante. C'est par exemple le stress, et non le trac, qui fait que quelques jours avant une grande mission, en particulier la dernière nuit, on ne dort en général pas très bien. Même si l'on s'aguerrit au fil des prestations et que l'on devient notamment beaucoup plus résistant à la fatigue, on ne peut pas dire que le stress diminue vraiment, du moins cela n'a pas été le cas pour moi.

Le trac, plus ponctuel, et aussi plus violent, est lié au fait que l'on est amené à paraître en public. Le stress fatigue, le trac fait souffrir.

En ce qui concerne le trac, il est banal de rappeler que de nombreux artistes célèbres en sont la proie avant d'entrer en scène(13). II s'agit donc d'un phénomène répandu, a priori inévitable et sans doute normal, dont il n'y a pas à avoir honte. II faut souligner qu'en règle générale il disparaît dès que l'on commence à travailler. C'est donc clairement un état qui a une limite dans le temps. Signalons également qu'il diminue d'intensité au fur et à mesure du déroulement de la mission, si elle dure plusieurs jours. II y a donc une accoutumance au trac. Mais elle n'est que provisoire, et il réapparaît à la mission suivante.

Dans la mesure où le stress et le trac peuvent handicaper l'interprète, parfois fortement, dans l'exercice de son rôle, il est important d'apprendre à leur résister.

Au cours de ma carrière, j'ai entendu dire que de nombreuses personnes ayant à prendre la parole en public prenaient préalablement un anxiolytique. Selon moi, cela n'est pas une bonne idée pour les interprètes, car cela risque d'émousser des facultés indispensables comme l'attention, ou l'aptitude à ressentir les choses, alors qu'au contraire l'interprète doit être au maximum de ces potentialités(14).

Par ailleurs, de nombreux artistes déclarent que s'ils n'avaient pas le trac, ils pensent qu'ils joueraient moins bien. Je suis d'accord avec cette opinion, appliquée cette fois à l'interprète. C'est précisément là le point crucial : il ne faut pas que le stress et le trac l'empêchent de faire son travail, mais au contraire le poussent à donner le meilleur de lui-même. II lui faut ainsi parvenir à " retourner " ce facteur psychologique qui peut faire obstacle, pour en faire une force motrice positive.

Comment faire ? Voici ma recette : j'ai toujours fait l'effort de considérer que cette personne qui allait travailler devant le public, moi-même, était, aux yeux des participants, des intervenants et du public, non pas moi-même, mais simplement l'interprète. II se trouve que c'était moi qui allais travailler, mais ce qui était important, c'était le rôle utile, et non pas la personne de l'interprète qui le remplissait.

(13) Pour les gens de ma génération, c'était le cas par exemple de l'humoriste Thierry Le Luron, l'incarnation même de l'aisance, de la décontraction et du brio.

(14) Mais une coupe de champagne (une seule) prise pendant le cocktail peut aider à se décontracter. 
Je me suis donc toujours mentalement " effacé " en tant que personne derrière ma fonction, ce qui m'a mis en état de bien la remplir, en me rappelant que sans interprétation, les interlocuteurs ne se comprendraient pas(15).

Enfin, il peut y avoir aussi des "techniques d'accompagnement " et, sans tomber dans l'obsession, rien n'empêche d'avoir un petit rituel qui permet de se rassurer : vérifier que l'on a bien tout son matériel, les coordonnées indispensables, les documents nécessaires, etc. En ce qui me concerne, je me souviens par exemple qu'à une époque j'avais l'impression de devenir un autre en chaussant mes lunettes, qu'elles faisaient de moi " l'interprète "...

En conclusion sur ce point, indiquons une dernière chose : quel que soit l'état psychologique dans lequel se trouve l'interprète, rien ne doit en transparaître à l'extérieur. II lui faut s'obliger à paraître parfaitement calme (si elles tremblent un peu, cacher ses mains, ou les poser bien à plat, etc.), ce qui a pour effet, paradoxalement, de calmer effectivement. Et il faut aussi penser à se forcer à continuer à respirer normalement...

\section{La gestion de la fatigue}

L'interprétation consécutive est en principe moins fatigante que l'interprétation simultanée. C'est la raison pour laquelle, alors que la simultanée se pratique toujours en équipe, avec un relais toutes les vingt minutes environ, l'interprète de consécutive peut être amené à travailler seul, dans les deux sens, et ce pendant toute la journée de travail et toute la mission. Même si cela est exceptionnel dans l'interprétation diplomatique, où l'on travaille en tandem, un interprète pour chaque délégation, les missions restent de toute façon épuisantes, du fait du décalage horaire(16), de la tension et de la fatigue physique due au travail et aux déplacements.

Le seul moment de vrai repos, c'est quand on regagne sa chambre une fois la journée terminée (encore faut-il prendre le temps de mettre à jour ses notes de la journée, et se préparer pour le jour suivant). Autrement, dans la journée proprement dite, il n'y a que de très rares pauses, il faut donc savoir les exploiter à fond.

Elles sont de trois ordres : les pauses aménagées pendant une réunion, les pauses correspondant aux déplacements, et enfin les pauses pendant les temps morts, les temps d'attente.

Les pauses pendant les réunions sont destinées à prendre un café ou un rafraîchissement, ou passer aux toilettes. Le problème est qu'à ces moments-là, même si les chefs s'absentent, les

(15) C'était vrai dans le cas des langues que je pratiquais. À ce propos, il faut signaler la difficulté supplémentaire que représente a contrario le fait d'interpréter dans des langues largement connues. II faut être excellent interprète pour que l'auditoire n'ait pas l'impression de perdre son temps alors qu'il a déjà bien compris un message.

(16) Je n'ai jamais trouvé de solution au décalage horaire. Je me suis toujours méfié des médicaments permettant de le combattre, et me suis résolu à en accepter les effets, tout en faisant le maximum pour récupérer chaque fois que possible. 
délégations restent en présence l'une de l'autre, les gens de part et d'autre vont se saluer, discuter, et bien souvent il y a encore besoin d'interpréter. La réunion est interrompue, mais l'interprète n'est pas en pause.

L'interprète doit alors veiller à pratiquer la rupture nécessaire, et ne pas laisser "filer " le temps de pause en se disant qu'il va y aller " dans une minute ". II faut donc éventuellement s'excuser et s'éclipser(17).

Dans les temps de transport, si l'on ne travaille pas, il faut là aussi se reposer le plus possible, et ne pas hésiter à fermer les yeux quelques minutes et s'octroyer un micro-repos. On s'aperçoit d'ailleurs rapidement que c'est ce que font tous les membres de la délégation au bout d'un ou deux jours de mission.

\section{La gestion des temps morts}

Compte tenu notamment des marges de sécurité prises, il y a toujours des temps d'attente(18), mais ces moments ne sont pas assez longs pour que l'on puisse véritablement s'absenter. La difficulté est alors de parvenir à "s'isoler " sur place, pour se relaxer sans se déconcentrer, tout en restant courtois avec les personnes environnantes. Les autres personnes qui attendent également viennent complimenter l'interprète (si tout va bien), lui poser des questions sur son métier, etc. L'interprète risque alors d'entrer dans une discussion qui, au final, contribuera pour beaucoup à la fatigue. Sans bien sûr paraître timide, ni distant, il faut parvenir à s'économiser dans une communication "normale " mais minimale avec l'entourage.

\section{L'économie}

Nous venons de parler des - rares - moyens permettant à l'interprète de se reposer. Mais la lutte contre la fatigue comporte un autre aspect : la réduction de la fatigue en cours de travail. La prestation, nous l'avons dit, implique un investissement fort et permanent. Cependant l'expérience permet d'apprendre à " doser " l'énergie investie, en évitant l'excès, en réduisant l'effort au strict minimum nécessaire. II ne s'agit pas ici de baisse de l'activité, ni bien sûr de sa qualité, mais d'optimisation de la dépense d'énergie. L'interprète travaille alors en quelque sorte en " mode économique " C'est ce facteur, que l'on peut qualifier de "sobriété dans la prestation ", qui permet sur le long cours de beaucoup moins se fatiguer lors des missions.

(17) II faut en profiter pour essayer de se relaxer au maximum. J'ai pour ma part observé que le simple fait de me laver les mains à l'eau chaude avait sur moi un effet relaxant.

(18) ... qui peuvent se terminer par de brusques accélérations du tempo, il faut faire attention à ne pas rater le passage de la personnalité ! 


\section{Le risque de la surexcitation}

Signalons enfin un dernier phénomène. En fait, au cours de la mission, l'organisme a naturellement tendance à s'adapter et à compenser la fatigue par des poussées d'adrénaline qui " survoltent " l'interprète, notamment pendant les temps forts de la mission. Une sorte d'exaltation s'installe alors, qui empêche de ressentir la fatigue, mais pousse par exemple en dehors du temps de travail à s'extérioriser beaucoup en bavardant avec les uns et les autres. C'est en quelque sorte un état de surexcitation euphorique inverse de la situation de trac et de stress. S'il est agréable, cet état n'en est pas moins pernicieux, parce qu'il incite l'interprète à continuer à se dépenser, alors même qu'il doit en permanence veiller à s'économiser. II faut donc être conscient de ce risque, et prévenir l'installation de cet état, ou au moins ne pas tomber dans le piège de la dispersion au moment de la pause ou pendant les temps morts.

\section{La fin de mission}

Pour conclure sur la gestion de la fatigue, disons quelques mots de la fin de la mission, du retour à la vie normale. La fin de la mission, c'est - on peut l'espérer - tout s'étant bien passé, le moment où l'on quitte le lieu de réunion, ou bien en récupérant ses bagages au retour, à l'arrivée à l'aéroport, le moment où l'on salue les collègues. Paradoxalement, c'est encore le début d'une phase délicate. 0 n vit alors à la fois la fin d'un épisode très intense, la disparition d'un objectif professionnel majeur, et conjointement un arrachement brutal au monde dans lequel on a évolué plusieurs jours, pour retomber dans les contingences quotidiennes, comme le fait de devoir prendre le métro alors qu'on a circulé plusieurs jours dans des cortèges.

II faut arriver à ménager une sorte de transition, une diminution graduelle de la tension, une décompression contrôlée, qui permet de se retrouver là encore avec le moins de déperdition possible dans l'état de la vie " de tous les jours", et non pas de rester en "surrégime " comme un moteur qui tournerait à vide, ou connaître un effondrement brutal accompagné d'une sensation de vide. C'est la dernière phase de la mission qui permet encore de faire des économies d'énergie... cette fois en vue de la mission suivante, et au fil des années sur l'ensemble d'une carrière.

Pendant les différentes phases de la mission, l'interprète est en permanence en contact avec différentes personnes associées à la visite. Nous allons en dire quelques mots.

\section{Les relations avec...}

\section{Le service du Protocole}

Nous avons évoqué le rôle du Protocole dans la préparation de la mission. Le Protocole intervient bien sûr de même tout au long de la réalisation de celle-ci, en en surveillant le bon 
déroulement dans les moindres détails. C'est notamment le Protocole qui s'occupe de la logistique complexe des cortèges de véhicules, et qui remet les badges donnant accès aux endroits réservés à la délégation ou à la personnalité.

\section{Le service des Voyages}

Pour tout déplacement d'importance, la logistique est assurée par le service des Voyages. L'interprète est pris en charge sur ce plan comme tous les autres membres de la délégation et, comme eux, est tenu de suivre les indications diffusées au début et au cours du voyage. L'interprète qui regagne sa chambre d'hôtel après une journée chargée doit encore vérifier qu'il est bien prêt pour le lendemain, et qu'il n'y a pas, quelque part à l'entrée ou sur le bureau, un pli contenant une information pratique importante, annonçant par exemple un changement dans l'horaire de départ du lendemain matin, ou dans l'heure de collecte des bagages " devant la porte " pour leur acheminement à l'étape suivante du voyage.

\section{Les proches collaborateurs de la personnalité}

Nous en avons déjà parlé à propos de la préparation de la mission. Ces personnes sont essentiellement les conseillers (mais aussi l'aide de camp qui porte les documents, discours, etc., et les transmet à la personnalité au moment où elle en a besoin). II est important que l'interprète les connaisse, pour pouvoir anticiper ce qu'elles peuvent être amenées à faire ou à dire, ou pouvoir lui-même les solliciter si besoin est.

\section{Les agents de sécurité}

Dans la mesure où ils gravitent de la même manière autour de la personnalité, en concourant à sa sécurité, nous classerons le ou les gardes du corps, chargés de la sécurité rapprochée et directement au contact de la personnalité, dans la même catégorie que les agents de sécurité, qui en sont un peu plus éloignés. II est évidemment fondamental que l'interprète évolue en bonne intelligence avec eux.

\section{Les journalistes}

Les journalistes sont, avec les hommes d'affaires, les personnes qui font partie de toute délégation de quelque ampleur. lls sont les autres personnes que l'interprète côtoie régulièrement, mais plutôt cette fois dans les " temps morts " de la mission. Ils connaissent très bien les dirigeants et la personnalité, dont ils sont même parfois proches. Leur métier est de glaner des informations, afin de les diffuser. L'interprète est une source d'informations potentielle. II doit donc faire comprendre d'emblée qu'il n'y a rien à attendre de sa part. Tout en faisant montre de la courtoisie de mise, il doit garder ses distances, et même dans les échanges a priori les plus anodins, doit faire montre de la plus extrême vigilance quant à la confidentialité. 
Je dois indiquer tout de suite qu'en fait, je n'ai jamais été vraiment sollicité par les journalistes lors des missions officielles auxquelles j'ai été associé. Peut-être aussi parce que mis en garde par les enseignants de l'ESIT (en particulier Christopher Thiéry), je me suis dès le début de mon activité montré très réservé et laconique dans ces contacts.

\section{La personnalité}

L'interprète n'est pas un proche de la personnalité, mais pendant la mission il en est proche. On pourrait penser que c'est impressionnant d'être aux côtés d'un ministre, du président de la République. Ça l'est, en réalité(19). Mais cela n'est que l'un des aspects "personnels " du travail de l'interprète, et ne doit pas interférer avec la réalisation de sa mission. On l'a dit à propos du trac, l'interprète doit "s'oublier en tant que personne ", mettre de côté ses propres réactions à la situation, pour se consacrer tout entier à son travail : servir la communication que la personnalité veut établir avec son interlocuteur étranger.

Cela étant dit, il y a néanmoins fort normalement une interaction " humaine " entre le " client " qu'est la personnalité et le " prestataire " qu'est l'interprète. Cette interaction est même une condition de la bonne réalisation de son travail, parce qu'il facilite la mise en confiance de la personnalité envers son interprète, ce qui est fondamental.

Cette interaction comporte en fait deux aspects, correspondant à deux moments distincts : pendant la traduction, et dans les moments précédant et suivant celle-ci. Pendant la traduction, il y a une proximité mentale qui s'installe, qui peut déboucher sur une sorte de sympathie et d'intimité intellectuelle(20). Même si l'interprète doit rester conscient des limites de cette proximité et de son caractère conjoncturel, car il n'est rien d'autre qu'un prestataire de service(21), elle contribue à faciliter la compréhension mutuelle et la transmission du message, objectif de l'interprétation. En marge de celle-ci, cette proximité se prolonge à travers le comportement de l'interprète vis-à-vis de la personnalité, à l'occasion de sa prestation pour elle.

L'interprète ne doit donc pas se fermer à ce type de contact pour faire son travail, il ne peut se cantonner dans la réserve. Sur ce plan, il ne peut pas rester neutre, au sens de "fermé " ou " indifférent ". Mais il doit trouver le " bon dosage ". On peut le qualifier comme suit : efficacité

(19) II peut même y avoir à la première rencontre un effet de sidération passagère, qui fait que l'on reste paralysé devant la personnalité.

(20) Le lecteur pourra lire à ce propos et sur les risques de malentendus que cela peut comporter l'article du Point (voir bibliographie) consacré à notre collègue anglophone Amanda Galsworthy, interprète présidentielle.

(21) Ce qui n'empêche en rien la personnalité d'éprouver respect et considération envers l'interprète. L'ouvrage publié par l'AllC à l'occasion de son 60e anniversaire (voir bibliographie) mentionne l'hommage discret que le Général de Gaulle a ainsi tenu à rendre à son interprète pour l'allemand, Jean Meyer (p. 106). J'y ajouterai la présence des autorités allemandes aux obsèques de Brigitte Sauzay, également longtemps interprète officielle, avant d'œuvrer aux relations franco-allemandes au-delà de cette seule fonction. 
dans la communication pendant l'interprétation, et en marge de celle-ci, prévenance envers la personnalité, comme on l'a dit à propos de sa sécurité physique, avec la courtoisie de mise, sans obséquiosité ni complication d'aucune sorte, comme si tout était normal et allait de soi.

Je soulignerai à ce propos le grand besoin de naturel et de simplicité que j'ai ressenti au fil du temps, en particulier chez les clients officiels : ils sont en permanence plongés dans un milieu qui cherche à leur plaire, à s'attirer leur faveur. Cela leur fait du bien d'avoir à leur côté quelqu'un simplement et naturellement à leur service.

Nous allons maintenant évoquer la place physique qu'occupe l'interprète pendant les temps d'interprétation à proprement parler.

\section{Le placement physique de l'interprète}

La question de l'endroit où est placé l'interprète pour travailler est très importante. La difficulté est que cet endroit varie avec les circonstances. Distinguons quelques cas.

Le plus classique d'abord : à la table de négociation. L'interprète sera de préférence assis à la table, immédiatement à côté du chef de la délégation, soit à sa droite, soit à sa gauche(22). Les proches collaborateurs du président de séance ont naturellement tendance à s'asseoir à ses côtés, et si sa place n'est pas prévue, l'interprète doit insister pour qu'on la lui cède. Si une autre personne est intercalée, une distance s'introduit, la personnalité est parfois masquée, l'audition est plus aléatoire, le risque d'erreur et la fatigue s'accroissent fortement.

II serait encore pire que l'interprète se trouve rejeté en bout de table, où cette fois ne se trouvant plus en face de son destinataire principal, il devrait faire un effort considérable pour que celui-ci l'entende bien ; même au cas où il dispose d'un micro, l'interprète ne verrait pas le destinataire, et donc ne saurait pas non plus s'il est bien compris de lui.

Les autres circonstances d'une mission officielle sont très nombreuses : dans les véhicules pendant les trajets, pendant les échanges au cours des déplacements à pied, pendant les réceptions, pendant les entretiens dans un salon, au cours des repas...

Dans un salon par exemple, il ne s'agit pas d'une véritable séance de travail à la table de négociation. Si l'on est en France à l'Élysée, les deux personnalités sont installées côte à côte sur un canapé(23), les quelques autres participants assis sur des fauteuils disposés en arc de cercle. L'interprète est à côté du président, sur une chaise que lui a approchée l'huissier.

(22) En fonction de ses propres préférences liées à sa " meilleure oreille ". J'ai le plus souvent été assis à la droite de la personnalité, me tournant légèrement vers la gauche pour l'écouter.

(23) Lors de la visite en France du Président chinois Xi Jinping fin mars 2014, le dispositif dans le salon de l'Élysée était différent : les deux présidents étaient chacun assis sur une chaise devant les drapeaux des deux pays. Mais peut-être s'agissait-il uniquement de la séance de photos (voir notre conclusion). 
Si l'on est en Chine, les personnalités sont assises dans un fauteuil, de part et d'autre d'une tablette basse sur laquelle une boisson sera servie. Les interprètes sont assis sur une chaise chacun immédiatement derrière "sa " personnalité. Les autres personnes sont assises dans des fauteuils disposés en " U ", Chinois d'un côté, Français de l'autre. Un petit micro est posé sur la table basse, à côté de chacun des interprètes. Les interprètes prennent des notes en posant leur carnet sur leurs genoux(24).

Pour rester en Chine, dans les grands banquets, pendant longtemps les deux interprètes étaient assis à table, chacun à côté de sa personnalité, les deux personnalités étant assises côte à côte, les autres interprètes étant répartis à table entre les convives. Par la suite, j'ai constaté un changement : lors des banquets avec des tables disposées en " U ", les interprètes étaient répartis à l'intérieur du U, les convives étant à l'extérieur. Cette dernière formule était de loin la plus pratique, offrant une excellente visibilité et beaucoup de place pour tous, convives, interprètes, et gens du service. Mais le dispositif a de nouveau évolué, et les interprètes se sont malheureusement retrouvés dans la même situation qu'en France.

En France, pendant les grands banquets, les interprètes sont le plus souvent assis derrière la personnalité, sur une chaise. Cela complique grandement la tâche de l'interprète, qui doit tendre le cou vers l'avant pour se faire entendre, tout en étant inconfortable pour la personnalité, obligée à l'inverse de se reculer. Cela complique enfin beaucoup la tâche des serveurs, qui doivent se pencher entre l'un et l'autre, pour servir et desservir(25).

À table pour les toasts, l'interprète se met debout à côté de la personnalité pour interpréter les passages successifs de son discours.

Pour l'interprétation des allocutions, s'il y a une tribune, l'interprète se trouve en général juste à côté de l'orateur ; s'il n'y en a pas, et qu'il y a simplement au centre de la pièce un micro sur pied, l'interprète est en général placé non loin de la personnalité, avec lui aussi un micro sur pied.

En résumé, la place qu'occupe l'interprète doit toujours être commode pour lui-même, vis-àvis de l'orateur, du destinataire et du public, avec lesquels il doit pouvoir toujours facilement entretenir le contact auditif et visuel. L'interprète occupe ainsi une place centrale, mais comme nous l'avons dit, il ne la doit qu'à son rôle.

Nous terminerons ces quelques lignes sur le placement physique de l'interprète par une remarque, sur laquelle nous conclurons ce petit tour dans les coulisses de la mission d'interprétation consécutive officielle.

(24) Ce dispositif, dont des photos anciennes montrent qu'il n'a pas changé depuis au moins cent ans, est assez pratique.

(25) C. Thiéry, dans l'article Interprétation diplomatique (p. 55), dénonçait déjà le recours à ce dispositif (voir bibliographie). 


\section{Conclusion : I'interprète et la photo}

Nous venons de le voir, le placement de l'interprète par rapport à la personnalité est variable, tout en répondant en permanence à certains impératifs. II est toutefois encore une circonstance différente, la présence de l'interprète " sur la photo ". II arrive en effet fréquemment que l'interprète officiel " passe à la télé " (où ses proches le guettent), et figure en arrière-plan sur de nombreuses photos des personnalités. Cela est normal lorsqu'il est en train d'interpréter, car il doit rester à proximité pour pouvoir faire son travail. Si les photographes ou les cameramen présents sont autorisés à officier, l'interprète n'a pas à s'en soucier. Mais dans les visites officielles, il y a des moments ménagés spécialement pour les photos.

C'est le cas par exemple avant le début d'un entretien : le Protocole fait entrer les photographes et les caméras dans le salon, en général en deux ou trois "vagues " pour éviter les bousculades. L'interprète est déjà en place, mais souvent ne travaille pas encore. II lui faut alors prendre une attitude naturelle, au cas où il serait à l'image.

Mais ce sont surtout l'arrivée et le départ de la délégation, au moment de l'accueil puis de la prise de congé, à un endroit symbolisant le lieu, par exemple sur le perron de l'Élysée ou des ministères. Un cordon est tendu derrière lequel se trouvent les photographes et cameramen, qui interpellent les personnalités et les mitraillent. À ce moment, lorsque les personnalités échangent une poignée de mains, ce qu'elles disent n'est plus que le prétexte à la prise de ces photos qui font la une des journaux, et l'interprète doit alors s'écarter hors du champ. Car pour le photographe et les personnes chargées de la communication publique des personnalités, la photo idéale est " propre ", sans détail importun, c'est-à-dire en l'occurrence sans l'interprète.

Si l'interprète doit alors encore interpréter quelques mots, il le fait de loin, en veillant à ne pas paraître à l'image. II s'efface devant le photographe et le cameraman, son rôle a pris fin.

wufaer@club-internet.fr 


\section{Bibliographie}

THIÉRY Christopher, 1990, " Interprétation diplomatique ", in LEDERER Marianne (textes réunis par), Directeur de l'ESIT, Université de Paris III, Études traductologiques, en hommage à Danica Seleskovitch, Paris, Lettres Modernes Minard, coll. Cahiers Champollion, p. 45-59.

( $C$. Thiéry décrit les différentes circonstances concrètes de cette forme d'interprétation, dont les modalités sont restées inchangées pour la partie interprétation consécutive.)

AllC, Groupe Histoire, 2013, Naissance d'une profession - Les soixante premières années de I'Association Internationale des Interprètes de Conférence (AlIC), Genève (Suisse), AllC éditeur.

(Cet ouvrage publié à l'occasion du 60e anniversaire de l'Association relate la genèse de l'interprétation moderne et l'organisation de la profession, puis présente de nombreuses notices biographiques ainsi que des rapports et décisions officiels. Un égal engagement et une grande liberté de ton au-delà de la diversité des styles. On y remarquera notamment la résistance opposée à la simultanée par les grands consécutivistes qui ont été les premiers à l'expérimenter. Ouvrage passionnant pour qui s'intéresse à l'interprétation de conférence.)

MAHRANE Saïd, 2013, "Elle a traduit Mitterrand, Chirac et Sarkozy ! ", in Le Bulletin de l'AAEESIT (Association des Anciens Élèves de l'ESIT), Bulletin $n^{\circ}$ 68, premier trimestre 2014, p. 7-8, 1 re parution in Le Point, décembre 2013.

(Cet article, consacré à notre collègue Amanda Galsworthy, interprète officielle français-anglais, montre le décalage entre la réalité vécue par les protagonistes et l'image renvoyée par les médias, ainsi que les malentendus qui peuvent résulter de la proximité entre la personnalité et l'interprète.) 